\title{
Keyword index to volume 10
}

$\beta$ globin gene cluster 320

$13 p$ translocation 467

$15 q 11-q 1426$

$16 q 24239$

3'-untranslated region 413

35 delG 427

$7 q 11250$

8p22-p23 18

$8 q 13.3757$

\section{$A B C A 4198$}

$A B C R 198$

ACCPN 406

ACHM3 638

Achondroplasia 819

Adenomatous polyposis coli (APC) 505

Admixture 530

Age estimation 439

Age-related hearing impairment 883

Agenesis corpus callosum 406

Aging 292

Amelogenesis imperfecta 865

Amyloidosis 786

Ancestors' nutrition 669

Ancestral haplotype 439

Androgen receptor 724

Aneuploidy 462

Angelman syndrome 26

Angiotensin-1 converting enzyme 553

Angiotensinogen 715

Anophthalmia 807

Antioxidants 539

ApoE concentration 841

ApoE polymorphism 841

ApoEurope 841

Apolipoprotein E gene 400

Apolipoproteins 292

Association 491, 562, 870

Association mapping 381

Association study 883

Asthma 82

Atherosclerosis 367, 539

Atopy 266

ATR-X 223

Austria 427

Autoimmune disease 475

Autosomal dominant disorder 757
AZF-Y chromosome 467

Azoospermia 362

\section{BAGE 833}

$B A X$ exclusion 95

Becker muscular dystrophy 413

Best vitelliform macular dystrophy 281

Bilirubin 539

Bipolar disorder 276

Bladder 819

BLK 18

Body mass index 749

BORIS 669

Bottleneck 381

BMP4 753

Branchio-Oto-Renal 757

BRCA2 6174delT 395

BRCA mutations 724

BRCA1 599

BRCA2 599, 879

BRCA2 testing 395

Breast cancer 724

Breast cancer susceptibility 599

British-Danish 381

Bronchial responsiveness 177

C677T mutation 388

CACNA1A 857

Calpain-3 825

Calpainopathy 825

Cancer 6, 819

Candidate gene 82

Candidate gene analysis 516

Cardiomyopathy 741

Cardiovascular disease 682

Cardiovascular risk 841

Carrier frequency 198

Cartilage-hair hypoplasia 439

Case-control study 82

Catatonia 491

Cationic trypsinogen gene (PRSS1) 100

Centenarian-only approach 119

Cerebral Cavernous Malformations 733

CF 231

CFTR 583

CFTR gene mutations 729

CHAC 773
Charcot-Marie-Tooth 297

Chemokine receptor cluster 52

Childhood overeating 682

Chorein 773

Choreoacanthocytosis 773

Chromatin structure 223

Chromosomal deletion 807

Chromosome 3807

Chromosome 3 deletions 52

Chromosome 6 259, 562

Chromosome 7177

Chromosome 9 210, 790

Chromosome 1262

Chromosome 13 266, 833

Chromosome 17p 271

Chromosome 19p13.1-13.2 162

Chromosome 21833

Chromosome Xp22.13516

Chronic recurrent multifocal osteomyelitis 217

Ciliary neurotrophic factor CNTF gene 749

Clarin 339

Cleavage stage embryos 801

Clinical variability 825

CMT1A 297

CNGB3 638

CNG channel 638

Coffin-Lowry syndrome 2

COL9A1 562

Colorectal cancer 499

Colour blindness 638

Combined segregation-linkage analysis 715

Complete achromatopsia 638

Complex disease 153, 313, 381

Complex I 141

Complex rearrangements 757

Compound heterozygotes 786

Cone-rod dystrophy 865

Congenital disorder of glycosylation 643

Congenital muscular dystrophy 91

Connexin 26 427, 495

Contiguous gene deletion syndrome 413

Coronary artery disease 539 
Cost-effectiveness 599

CpG island 6

CRMO 217

Cryptic rearrangements 707

CSNB2 449

CTG18.1 276

CYP21 gene 137

Cystic fibrosis 231, 583

Cystic fibrosis transmembrane conductance regulator (CFTR) 100

Cytogenetics 467

\section{D18S60 217}

D19S246 95

De novo mutation 213

De novo mutations 631

Deafness 339, 391, 421, 851

Deletion 72

Deletion/duplication 26

Demographic variables 689

DFNA5 883

DFNB33 391

DHPLC 643

Diabetes 669

Diabetes mellitus 421, 682

Diagnosis 2

Digenic 72

Disruption of elastin 351

DNA methylation 6

DNA pooling 125, 870

DNA repeat 499

Dosage compensation 44

Down syndrome 388

Duchenne muscular dystrophy 413

Duplicons/segmental duplications 26

Dyslipidaemia 547

Dystrophin 413

DYT1 gene 213

Elimination test 52

EMD 505

Emerin 157

Emery-Dreifuss muscular distrophy 157

Encephalomyopathy 289

Enzymatic mutation detection 505

EPHX 569

Epidemiology 583

Epididymal sperm aspiration 362

Epigenetic inheritance 669

ERDA1 276

Ethnicity 583

Exclusion 250

Exclusion testing 591

Experimental errors 125

Extracellular matrix 91
EYA1 757

\section{FAM11A 767}

FAM11B 767

Familial adenomatous polyposis 631

Familial balanced translocation 351

Familial Mediterranean fever (FMF) 145, 786

Family based association study 217

Family Heart Study 367

Family planning 168

Family study 729

FAP 505

FBN1 673

Female infertility 303

Fibrillin-1 673

Fibroblast growth factor receptor 3 (FGFR3) 819

Fine mapping 250

FISH 467, 790, 807

FMF carriers 786

Foetal 113

Folate 113

Founder effect 666

Founder mutation 198, 879

Founder population 406

Four-transmembrane-domain proteins 339

Fragile sites 767

FRAXF 767

Frontotemporal dementia 400

FYVE domain 52

Gaucher disease 511

Gene based quantitative fluorescent PCR 462

Gene expression 62

Gene family 833

Gene fragment 833

Gene localisation 210

Gene-gene interaction 119

Genetic association 400

Genetic basis 217

Genetic counselling 82, 297

Genetic map 177

Genetic mapping 516

Genetic testing 599

Genetics 82, 303, 367, 475, 539, 658, 715,741

Genomic imprinting 6

Genomic instability 499

Genomic screen 271

Genotype $\times$ phenotype correlation 145 , 334,825

Genotype-phenotype correlations 511
Genotyping 188, 870

Genotyping error 616

Germline mutation 457

GJB2 72, 495

GJB2 gene 391

GJB6 72

Glial cell line-derived neurotrophic factor 183

Glycerol kinase 413

Glycine 188

Glycosylation defects 643

Handedness 623

Haploinsufficiency 733

Haplotype 616, 857

Haplotype analysis 313

Haplotype mapping 553

Haplotype sharing 250, 271

HDL cholesterol 367

Hearing disorder 95

Hearing loss 851

Hepatocellular carcinoma 239

Hereditary breast cancer 395

Hereditary breast/ovarian cancer 879

Heteditary 213

Heteromorphism 790

Heteroplasmy 141

High resolution mapping 607

Hirschsprung disease 183

HLA 259

Homocysteine 113, 433

Homozygosity mapping 210

HPFH 320

Human deafness 210

Human interstitial telomere 107

Human leukocyte antigens 327

Human longevity 292

Huntington's disease 168, 591, 689

Hyperekplexia 188

Hypermutable 505

Hyperphenylalaninaemia 530

Hypertriglyceridaemia 547

Hypertrophy 741

Hypochondroplasia 819

Hypophosphatasia 666

Idiopathic chronic pancreatitis (ICP) 100

Idiopathic generalised epilepsy (IGE) 857

$\lg \mathrm{E} 266$

$I_{\text {Kur }} 36$

IL1RAPL1 413

Immuno-FISH 107

Imprinting 669, 694

Infertility 790 
Inherited cardiac conduction disorders 36

Insulin-like growth factor 1 receptor gene 699

Inter-individual lipid levels variation 841

Intermediate allele 204

Intermediate phenotype 715

Interruption 204

Intracytoplasmic sperm injection 362

inv dup(15) 26

Jordan 391

Juxtacentromeric regions 833

\section{KCNA7 36}

kcna7 36

Keratolytic winter erythema 18

Klinefelter syndrome 137

Krit1 733

KV1.7 36

KWE 18

Laminin 91

Lamins A/C 157

Left-handedness 623

Leigh disease 141

Leucine rich repeat domain (LRR) 52

Leukaemia 62

LGMD2A 825

Limb-girdle muscular dystrophy type $2 \mathrm{~A}$ 825

Linkage 162, 259, 475, 562, 870

Linkage analysis 95, 327, 375, 539, 623

Linkage disequilibrium 313, 406, 616, 857

Linkage disequilibrium mapping 607

Linkage mapping 607

LOH 239

Longevity 119

Low HDL cholesterol 547

Lysosomal storage disorders 813

Macrocephaly 699

Major histocompatibility complex 327

MALDI-TOF-MS 188

Male infertility 303

Marfan syndrome 673

MECP2 487

MECP2 gene 484

MECP2 77, 86

MEFV 145

MEHMO 226

Meiosis 467, 801

Mental retardation 82, 223, 487

Merosin-deficiency 91
Meta-analysis 400

Methylenetetrahydrofolate reductase 113

MFS 673

Microcephaly 807

Microphthalmia 807

Migration 530

Miller-Dieker syndrome 707

Mitochondria 289

Mitochondrial DNA 141

Mitochondrial mutation 851

MLC1 491

Modifier genes 724

Molecular beacons 427

Monte Carlo methods 327

Mortality 682

MPI 643

mtDNA 226, 521, 851

MTHFR 113

MTHFR gene 388

Multicolour banding (MCB) 790

Multifactorial disease 658

Multiple sclerosis 271

Multiple sulphatase deficiency 813

Multiplex fluorescent PCR 231

Mutation 141, 334, 421, 427, 439, 484, 491, 530, 583, 649, 819

Mutation analysis 100, 511, 638, 643

Mutation detection 757

Mutation frequency 487

Mutation screening 449

Mutational spectrum 773

Mutations 2, 303

Myosin light chains 741

Nance-Horan syndrome 516

Neural tube defects 433, 753

Neuroacanthocytosis 773

Neurofibromastosis type 1334

NOG 753

Nondisjunction 694

Non-penetrance 213

Non-syndromic 391

Non syndromic deafness 72

Norway 521

Nuclear envelope 157

Ophthalmogenetics 449

Osteoarthritis 562

Overgrowth 699

Pancreatic secretory trypsin inhibitor (PSTI/SPINK1) 100
Paraganglioma 289, 457

Parent of origin effects 177

Parental origin 631

Penetrance 724

PFHB1 36

PGD 231, 801

Phaeochromocytoma 457

Phaeochromocytomas 289

Phenylalanine hydroxylase 530

Phenylketonuria 530

Physical map 18, 266

Pigmentosa 245

Pim-1 245

PMM2 643

Polymorphism 82, 113, 292, 320, 421, 499, 569

Population 521

Population association study 749

Population genetics 153, 666

Porphyria 649

Porphyrin 649

Portwine stain 375

Position effect 351

Postaxial polydactyly 162

Potassium channels 36

Power 133

Power study 313

Prader-Willi syndrome 26

Pre-implantation genetic diagnosis 231

Predictive testing 168

Preeclampsia 569

Preimplantation genetic diagnosis 168, 297, 591

Prelingual deafness 495

Prenatal 297

Prenatal diagnosis 694

Prenatal testing 168, 689

Primary torsion dystonia 213

Pristane-induced arthritis 475

Promoter 499

Protein truncation test 505

Proteins 107

Psoriasis 327

QTL 475, 553, 607

QTL association analyses 125

Quantitative trait locus (QTL) 870

Race 583

Random genetic drift 381

Rats 475

Receptor 188

Recessive 391

Reciprocal translocation 801

Recominbation analysis 406 
Recurrent miscarriages 707

Repeat expansion 204

Reproductive decision making 168

Respiratory chain 226

RET proto-oncogene 183

RET activation assay 183

Retina 865

Retinal degeneration 865

Retinal dystrophies 198

Retinitis 245

Retinitis pigmentosa 339

Rett syndrome 77, 86, 484

Review 673

RFP-TM protein family 281

Rheumatoid arthritis 475

Ring X 44

RMRP 439

RP9 245

RSK2 2

SADDAN 819

Sample selection 125

Sarcoidosis 729

Satellite DNA 790

SCA1 204

Schizophrenia 491

Screening of mutation 825

SDHD 457

Segregation 801

Segregation analysis 327, 367, 547

Selenoprotein P 499

Sensorineural deafness 427

Serum IgE 658

Sex chromosomes 137

Shwachman-Diamond syndrome 250

Simulation 133

Single-cell PCR 591

Single-nucleotide polymorphism (SNP) 82,870

Skeletal muscle 91
Slovenia 879

Slow growth period 682

Small dense LDL 547

SNP 313, 616, 883

SNP genotyping 153

SNPs 125,857

Somatic mosaicism 77

Sperm aneuploidy rate 362

Spermatogenesis 467

Spina bifida aperta 753

Spinal neurofibromatosis 334

Spontaneous abortion 113

Startle disease 188

Steroid 21-hydroxylase 137

Steroid 21-hydroxylase deficiency 137

STGD 198

Stratification 259

STRs 297

Structure 673

Subtelomere FISH 707

Succinate dehydrogenase 289

Sudden cardiac death 741

Sulphatases 813

Susceptibility gene 271

T7511C 851

Taiwanese 495

TDT 266

Teeth 865

Testicular sperm aspiration 362

Testicular sperm extraction 362

TIN2 107

TPST1 gene 250

Trans-heterozygote 100

Transcobalamin 433

Transcribed retropseudogene 767

Transcript map 18

Transcriptional silencing 767

Transcriptional units 62

Transgenerational effects 682
TRF2 107

Triplet repeat expansion 276

Trisomy 15q26.1-qter 699

Trisomy 20 mosaicism 694

Tumour 833

Tumour necrosis factor alpha 82

Tumour suppressor gene 62

Tumour suppressor genes 52

Turner syndrome 44, 137

Type I error 133

Uncultured amniocytes 462

Uniparental disomy 694

Untranslated gene 439

USH3 339

Variable expression 351

Variance components approach 133

Variants 505

Variation 153

Vascular malformation 375

Vitamin B12 433

VMD2 281

Voltage-gated calcium channel 857

WFDC1 239

WFS1 421

Williams-Beuren syndrome 351

WKL1 491

Wolfram syndrome 421

$X$ chromosome inactivation 44

X-linked mental retardation 226

XIST 44

XNP 223

Y chromosome 521

Yp 467 